\title{
The Influence of Perivascular Adipose Tissue on Vascular Function in a Rabbit Model
}

\author{
Abdulqader Abdulrazzaq, Simon Kennedy \\ Institute of Cardiovascular \& Medical Sciences, University of Glasgow, Glasgow, UK \\ Email: alhadithi_92@hotmail.com
}

How to cite this paper: Abdulrazzaq, A. and Kennedy, S. (2020) The Influence of Perivascular Adipose Tissue on Vascular Function in a Rabbit Model. Pharmacology \& Pharmacy, 11, 331-349. https://doi.org/10.4236/pp.2020.1112027

Received: November 12, 2020

Accepted: December 13, 2020

Published: December 16, 2020

Copyright (c) 2020 by author(s) and Scientific Research Publishing Inc. This work is licensed under the Creative Commons Attribution International License (CC BY 4.0).

http://creativecommons.org/licenses/by/4.0/

\section{(c) (i) Open Access}

\begin{abstract}
The recent acknowledgment of the paracrine role of perivascular adipose tissue (PVAT) in vascular modulation has been supported by many studies investigating major arteries in several animal models and humans. The influence of PVAT on the functional activity of the vascular bed has been a matter of debate, whether it is an anticontractile effect with protective roles or a pro-contractile effect, investigations are underway to address this obscurity. In this investigation, we have studied the effects of vasoconstrictors, phenylephrine and noradrenaline, and vasorelaxants, carbachol and s-nitroso-nacetylpenicillamine (SNAP), on subclavian and iliac rings with and without PVAT attached; and concentration-response curves were constructed accordingly. Levels of nitric oxide (NO) generated due to activation of the enzyme adenosine monophosphate-activated protein kinase (AMPK) by 5-Aminoimidazole-4-carboxamide-1- $\beta$-D-ribofuranoside (AICAR) were measured in subclavian and iliac PVAT conditioned media. Additionally, Haematoxylin and Eosin staining was performed to analyze and compare the histological characteristics of both arteries. Subclavian and iliac rings with PVAT attached showed stronger contraction to phenylephrine and noradrenaline than that of rings without PVAT attached. At the same time, relaxation tests reported lower relaxation percentages in subclavian and iliac rings with PVAT attached compared to rings without PVAT attached in response to carbachol and SNAP. PVAT treated with AICAR generated higher levels of NO compared to levels of untreated PVAT. Conclusions drawn were the pro-contractile effects demonstrated by the PVAT especially in high concentrations of drugs used. In addition, histology analysis revealed characteristics of white adipose tissue in both PVATs.
\end{abstract}

\section{Keywords}

Perivascular Adipose Tissue, Nitric Oxide, Myography 


\section{Introduction}

The perivascular adipose tissue (PVAT) is becoming an important part of the cardiovascular system that plays a significant role in vascular modulation. It possesses paracrine signaling that influences the vasculature, and therefore the vascular function. Described as "The sixth man of the cardiovascular system" by Cheng et al. [1], the PVAT is not merely a mechanical support as believed in the past, but is an endocrine organ that regulates and modifies the vascular tone through communication with incoming neuronal signals, interaction with inflammatory mediators, and as a secreting entity that causes contraction/relaxation of the vascular wall. These capabilities make it possible that PVAT will emerge as a potential therapeutic target in controlling vascular responses to different constricting and relaxing drugs, in addition to its possible participation in the underlying causes of atherosclerosis, diabetes mellitus, and cardiovascular diseases. Figure 1 illustrates the anatomical position of PVAT in an artery.

Typically, the adipose tissue is composed of three types of adipocytes; white, brown, and beige depending on its region [2]. White adipose tissue (WAT) is characterized by its white and large adipocytes that function typically as energy storage. Brown adipose tissue (BAT) is termed so due to the high number of mitochondria that makes it recognizable by its metabolic activity and smaller adipocyte size. The mitochondrial content gives the characteristic brown color of the BAT that is heavily innervated to respond to sympathetic impulses to generate heat and energy [3]. Although it is less well innervated than BAT, WAT is distributed throughout different tissue depots that store fatty acid molecules as triglycerides and these surround multiple organs to form visceral fat and distribute

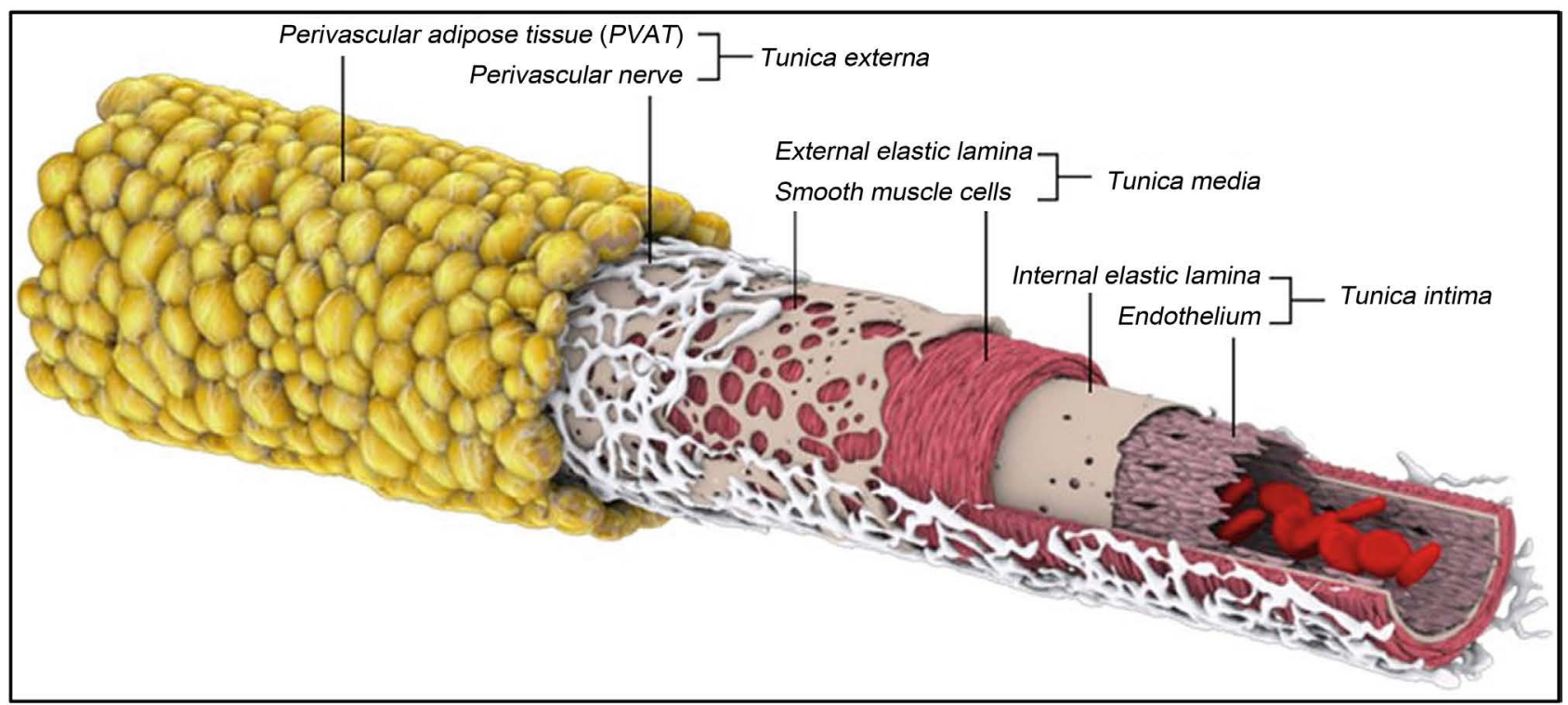

Figure 1. The PVAT is positioned in the external layer of the artery (Tunica externa, also known as Tunica adventitia), where it retains the physical function of protecting the artery in addition to its paracrine properties that influence the arterial tone. The perivascular nerve supply is attached to the PVAT and the rest of the artery allowing interpretation of neuronal impulses by PVAT, VSMCs and endothelium for an action to be concluded [14]. 
in subcutaneous areas. The low mitochondrial content is accredited to its energy saving capacity and mechanical support for the body. Moreover, WAT contains beige adipocytes that are also called brown-like adipocytes, which differ from BAT in that they contain lower expression of uncoupling protein 1 (UCP1). Also called Thermogenin, UCP1 is most abundant in the mitochondria of the BAT. It contributes to nonshivering thermogenesis, a process by which the BAT generates heat [2].

Interestingly, the PVAT is composed of both WAT and BAT depending on its location in the body. Studies have shown that PVAT phenotype is related to its anatomical position in the body; however, in pathological conditions, the increased levels of Angiotensin II and pro-atherosclerotic factors leads to dedifferentiation of adipocytes in PVAT [3]. In addition, recent studies demonstrated that mice exposed to chronic cold or continuous adrenergic stimulation can lead to conversion of WAT to brown or beige in a process called "Beiging of WAT", which favors breaking down, rather than storing, triglycerides in lipid droplets [2] [4]. This is supported by the functional similarities such as thermogenesis and energy production seen in both brown PVAT and BAT in cold temperatures [5]. Generally, the PVAT in the iliac vessel displays an equal amount of brown and white adipocytes, while PVAT surrounding the subclavian artery appears to be brown, which are the two vessels adopted in this investigation using rabbits [4] [6].

With differences in adipocyte color and expression level of UCP1 in PVAT, its effect on arterial vasculature remain to be the topic of recent studies. Its activity as an endocrine and paracrine organ has been established as it produces many substances that influence the underlying vascular smooth muscle cells (VSMCs) in major arteries. PVAT, along with the endothelium, are the pathways that modulate the vascular tone, which can have detrimental effects on vascular efficiency in pathological conditions. The adipose tissue within the PVAT secretes biologically active molecules that can be classified into adipokines such as adiponectin, leptin, resistin, and visfatin; cytokines such as tumor necrosis factor- $\alpha$ (TNF- $\alpha$ ) and interleukins; and nitric oxide (NO) and hydrogen sulfide $\left(\mathrm{H}_{2} \mathrm{~S}\right)$, which both identify as gaseous molecules that play critical role in initiating relaxation in the VSMCs. PVAT is a source of reactive oxygen species like superoxide and hydrogen peroxide $\left(\mathrm{H}_{2} \mathrm{O}_{2}\right)$, which play pivotal roles in contraction and relaxation of VSMCs respectively [1] [3] [7].

Categorically, some of the released molecules can have either contractile or anticontractile effects. Adiponectin, $\mathrm{H}_{2} \mathrm{~S}$, and angiotensin $1-7$ have been suggested to be relaxing factors that activate calcium-dependent $\left(\mathrm{K}_{\mathrm{Ca}}\right)$ potassium channels in VSMCs, which causes relaxation [5]. Typically, there are two mechanisms through which the PVAT exerts its anti-contractile effects. The endothelium-dependent relaxation, which activates endothelial nitric oxide synthase (eNOS) to synthesize NO that ultimately causes opening of $\mathrm{K}_{\mathrm{Ca}}$ channels by stimulating soluble guanylyl cyclase (sGC) to produce cyclic guanosine monophos- 
phate (cGMP), and the endothelium-independent relaxation that involves the production of $\mathrm{H}_{2} \mathrm{O}_{2}$, which either activates eNOS or directly causes relaxation through hyperpolarization of $\mathrm{K}_{\mathrm{Ca}}$ channels [5] [8] [9]. Either way, the $\mathrm{NO}$ and $\mathrm{H}_{2} \mathrm{O}_{2}$ are critical in transforming relaxation signals to actual vasodilation, but the molecular process that leads to their activation by adipokines and other PVATderived substances remain elusive.

One proposed pathway which acquired the attention of many of the studies concerned with vascular molecular interactions is the activation of adenosine monophosphate-activated protein kinase (AMPK) by different PVAT-derived relaxing factors like adiponectin [4]. In a recent study, it was hypothesized that AMPK activates eNOS by phosphorylation. Aortic PVAT-containing rings from AMPK knock-out (KO) mice were compared with the wild type (WT) in terms of response to contractile and relaxant agents. In the $\mathrm{KO}$ mice, the contraction response to U46619 was significantly higher than in WT mice; moreover, rings from $\mathrm{KO}$ mice had reduced relaxation in response to cromakalim; therefore the reduced anti-contractile effects of PVAT are attributed to the diminished content of AMPK, which in WT mice is activated by phosphorylation due to adiponectin secretion by PVAT [1] [8]. Because of the apparent importance of AMPK in our investigation, we have studied the effect of 5-Aminoimidazole4-carboxamide-1- $\beta$-D-ribofuranoside (AICAR), an AMPK activator, on the production of NO from PVAT taken from subclavian and iliac arteries of rabbits.

PVAT can also produce molecules that possess contractile effects such as angiotensin II and superoxide [8]. In rat mesenteric artery, the release of angiotensin II by the PVAT plays a critical role in promoting electrical field stimulation (EFS)-mediated contraction, which suggests that physiological neuronal stimulation enhances the constricting effect of angiotensin II. Moreover, treatment with an angiotensin I-converting enzyme inhibitor (ACEI) enalaprilat and angiotensin II type I receptor blocker (ARB) candesartan diminished the EFS-mediated contraction in arteries with PVAT attached, while arteries with removed PVAT were unaffected proving that the PVAT is the actual source of angiotensin II [10]. Takemori et al. [11] also showed that blood vessels of genetically modified lipoatrophic mice that suffer from loss of adipose tissue have contractile behavior that subsequently leads to development of hypertension, and that this pro-contractility is attributed to increased expression of vascular angiotensin II type I receptors. This study also suggests that PVAT is a contributing factor in maintaining normal levels of angiotensin II; as the vascular demand, portrayed by number of receptors expressed, for angiotensin II has increased due to PVAT absence.

In small arteries taken from visceral fat of obese patients, the contractile effect of PVAT was evident in the increased production of TNF- $\alpha$, which reduced NO bioavailability due to the production of superoxide by activating nicotinamide adenine dinucleotide phosphate (NADPH) oxidase and inducible NOS (iNOS), and inhibition of eNOS [12]. Suggesting that obesity-induced inflammatory activity supports pro-contractile effects of PVAT and a further study of how this is 
involved in cardiovascular complications is warranted [13]. Additionally, superoxide can also be produced by the NADPH oxidase-stimulating activity of angiotensin II, which then induces the activation of tyrosine kinase and subsequent activation of MAPK/ERK1/2 pathway; thereby emphasizing the involvement of the renin-angiotensin system (RAS) in influencing the paracrine function of PVAT [10].

In pathological conditions such as obesity, atherosclerosis, cardiovascular and metabolic diseases, PVAT secretion balance is disturbed. For example, its content of immune cells and chemokines is upregulated, which creates an inflammatory state that increases proliferation and activation of macrophages and $\mathrm{T}$ lymphocytes, which in turn reduces the release and anti-inflammatory and dilatory effects of protective adipokines, and favors the release of contractile agents and pro-inflammatory adipokines such as TNF- $\alpha$ and interleukin-6 [13]. Consequently, dysfunctional PVAT can have a detrimental impact on the production and activation of NO. NO content in PVAT is of interest and has been measured in the current research reported here, and it may play a critical role in development of pathophysiological conditions.

The consideration of PVAT as a therapeutic target has been growing recently with studies showing evident paracrine roles and unknown participation in many physiological pathways that are yet to be established. One is the autonomic innervation of PVAT, which is not fully understood and which may play an important role in defining the secretion profile of the PVAT that influences vascular function; secondly, the regional differences in PVAT adipocytes, whether their color or level of differentiation, may have effects on secretion capacity of certain adipokines that in turn affects the total function and participation of PVAT in vascular modulation. With the gained knowledge of signaling pathways and the variant adipocyte phenotypes in different regions of the body, PVAT-derived adipokines can be considered as targets and biomarkers in therapeutic and diagnostic measures of cardiovascular and metabolic disorders.

The aim of this investigation is to study the effects of phenylephrine and noradrenaline as constrictors, and carbachol and S-Nitroso-N-Acetylpenicillamine (SNAP) as relaxers in rings with and without PVAT taken from subclavian and iliac arteries of rabbits using wire myography. Moreover, an NO analyzer (NOA) was used to measure NO concentrations in PVAT conditioned media treated with AICAR compared to untreated media; as AMPK is suspected to mediate anticontractile effects of PVAT, NO concentrations in treated media are expected to rise as opposed to untreated media. In addition, visualizing and identifying adipose tissue in PVAT to understand the association of increased UCP-1 expression in brown PVAT to its adipokine secretion profile that influence the vascular tone.

\section{Methodology}

\subsection{Animal Model and Artery Preparation}

Rabbits used in this study were 18-week-old New Zealand White males. They 
were provided by Envigo and maintained at ambient temperatures. Animals were euthanized using $1 \mathrm{ml}$ of $200 \mathrm{mg} / \mathrm{ml}$ phenobarbital (IV) injection into the marginal ear vein. Subclavian and iliac arteries were dissected and kept in cold oxygenated (95\%: $\mathrm{O}_{2}: 5 \% \mathrm{CO}_{2}$ ) Krebs solution, also known as Physiological Salt Solution (PSS), which is prepared using the composition: $119 \mathrm{mM} \mathrm{NaCl}, 4.7 \mathrm{mM}$ $\mathrm{KCl}, 1.2 \mathrm{mM} \mathrm{MgSO}_{4}, 24.9 \mathrm{mM} \mathrm{NaHCO}_{3}, 1.2 \mathrm{mM} \mathrm{KH}_{2} \mathrm{PO}_{4}, 11.1 \mathrm{mM}$ Glucose and $2.5 \mathrm{mM} \mathrm{CaCl}_{2}$, and stored at $2^{\circ} \mathrm{C}-8^{\circ} \mathrm{C}$ for up to a week. Arteries were then isolated from surrounding tissue and sectioned into $2 \mathrm{~mm}$ long rings with and without PVAT attached.

\subsection{Large Vessel Wire Myography}

The rings were mounted on a 4-channel DMT wire myograph. Each channel is composed of a temperature-regulated bath with suction mechanism, gas supply and pair of pins. One pin is attached to a micrometer to allow control of vessel circumference, and the other is attached to a force transducer that measures the tension caused by the vessel in response to certain compounds. The baths were filled with $5 \mathrm{ml}$ of cold Krebs solution with the gas valve opened to sustain oxygen supply for the vessel. Rings were then mounted on the pins carefully under the microscope to ensure the endothelium remains undamaged during the process. Vessels were then stretched to 9.8 Millinewtons $(\mathrm{mN})$, which is equivalent to 1 gram of force, followed by turning the heating system on with temperature set to $37.5^{\circ} \mathrm{C}$ and washing baths with warm Krebs solution; then vessels were allowed to stabilize for at least 30 minutes. After stabilization, transducers were zeroed to set $9.8 \mathrm{mN}$ as the baseline. Figure 2 shows two mounted rings.

Baths were emptied and filled with $5 \mathrm{ml}$ of warm $62.5 \mathrm{mM}$ of KPSS, which is a high potassium-containing PSS, of the composition: $1.2 \mathrm{mM} \mathrm{MgSO}_{4}, 62.5 \mathrm{mM}$

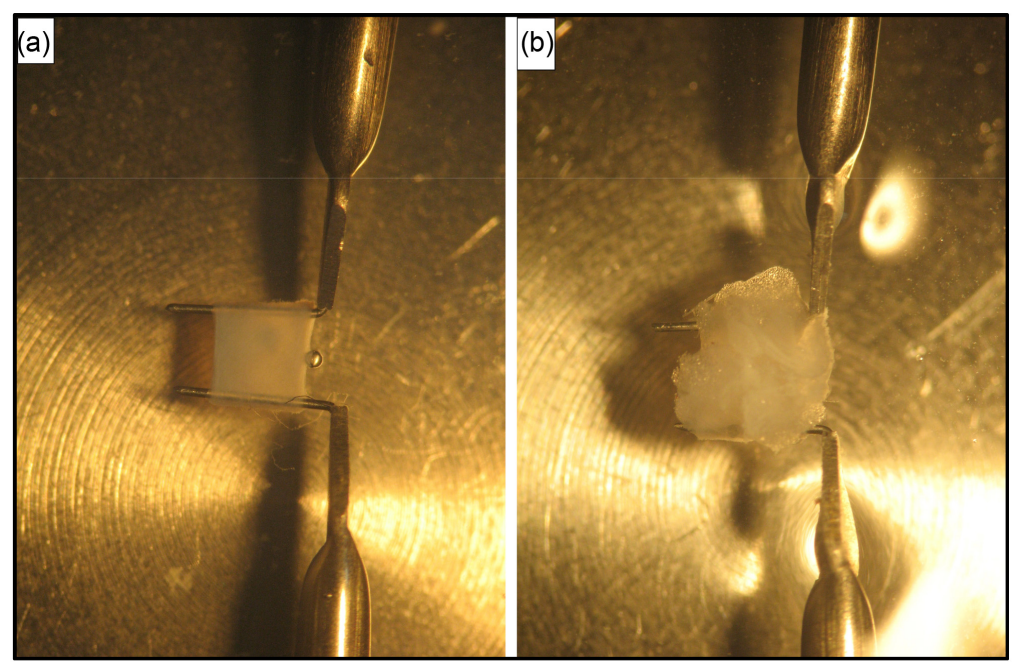

Figure 2. (a) shows a ring with no PVAT attached mounted on the pins of the myograph and stretched to $9.8 \mathrm{mN}$, while (b) shows a ring with intact PVAT in a similar position. 
$\mathrm{KCl}, 24.9 \mathrm{mM} \mathrm{NaHCO} 3,1.2 \mathrm{mM} \mathrm{KH}_{2} \mathrm{PO}_{4}, 2.5 \mathrm{mM} \mathrm{CaCl}_{2}$ and $11.1 \mathrm{mM}$ Glucose, to stimulate the vessels to "wake up" after a period of inactivity. Solution was left for 5 minutes, then washed with PSS three times to allow steady return to baseline. Subsequent addition of KPSS stimulates vessels to generate maximum contraction in response to extreme depolarization of VSMCs by the high amount of potassium followed by three-time washing with PSS to return the vessel to baseline for addition of vasoconstrictors.

\subsection{Endothelial Check and Cumulative Dosing}

Testing the endothelial function and integrity was conducted using a single dose of acetylcholine to give concentration of $1 \times 10^{-6} \mathrm{M}$ in the organ baths after the arteries were pre-contracted using a single dose (concentration ranging from $1 \times$ $10^{-6}$ to $1 \times 10^{-5} \mathrm{M}$ ) of either phenylephrine or noradrenaline depending on the ring's responsiveness to the dosage. A minimum relaxation of $50 \%$ or more was considered adequate for the endothelium to be deemed functional; as cholinergic receptors are situated on the surface of the endothelial cell that activate relaxation cascade through NO. Thereafter, baths were washed with PSS three times and left to stabilize to baseline.

Stable rings, with and without PVAT attached, were tested with cumulative doses of vasoconstrictor and relaxant agents. Average response to phenylephrine and noradrenaline as contracting agents was calculated and compared. Doses were added at five-minute intervals to yield concentrations starting from $1 \times 10^{-9} \mathrm{M}$ progressing to $3 \times 10^{-5} \mathrm{M}$. Concentration-response curves were constructed to identify PVAT influence on vessel contractility.

On the other hand, pre-contracted rings responses to SNAP and carbachol as relaxants were measured as percentage relaxation. Rings were pre-contracted with various submaximal doses of phenylephrine to generate sustained and submaximal contraction as baseline for relaxation data. Addition of relaxant was at five-minute intervals and concentrations ranged from $1 \times 10^{-9} \mathrm{M}$ to $1 \times 10^{-5}$ M.

\subsection{PVAT Conditioned Media Preparation and Nitric Oxide Quantification}

Measurement of PVAT-derived nitric oxide concentrations in conditioned media was conducted under the influence of AICAR, which is an AMPK activator, and compared with control media to examine the anti-contractile effect of AMPK and its involvement in NO production. PVAT was isolated from both arteries, weighed and divided equally in Eppendorf tubes with cold PSS solution. Tubes were then placed in distilled water baths set to $37^{\circ} \mathrm{C}$ with gas supply to warm up for 10 minutes before addition of AICAR. Dose was added to yield a concentration of $1 \times 10^{-3} \mathrm{M}$ and allowed to incubate for 30 minutes before removal of PVAT for snap freezing using liquid nitrogen. Thereafter, $100 \mu$ of the media was extracted and diluted with methanol at 1:5 and centrifuged at $17 \times \mathrm{g}$ for 20 
minutes at $4^{\circ} \mathrm{C}$ temperature, while the remaining $900 \mu \mathrm{l}$ of the media was stored at $-80^{\circ} \mathrm{C}$. Supernatant was collected to eliminate potential contaminant peptides for NO quantification as peptides lead to formation of foam in the purge vessel of the NOA, which affects the measurements of NO concentrations.

Measurement of NO concentrations was performed using Sievers 280 NOA, which measures $\mathrm{NO}$ in the chemiluminescence reaction cell. The NO secreted from the PVAT is oxidized in the conditioned media to produce nitrite; for this reason, the NOA is connected to a radical purger that contains a reducing agent ( $1 \% \mathrm{wt} / \mathrm{vol}$ of $\mathrm{NaI}$ in acetic acid) and a nitrogen gas supply to convert nitrite back to NO for measurement by the NOA. After calibration of NOA using a range of concentrations of sodium nitrite $\left(\mathrm{NaNO}_{2}\right)$, the supernatant was injected into the purge vessel in duplicates and their average was taken as the final NO concentration in the supernatant. Lastly, blank media was prepared using only the PSS solution to eliminate potential nitrite contamination within the media. The final concentration was adjusted for the blank, the dilution with methanol at 1:5 and the PVAT weight to calculate the amount of NO secreted by one milligram of PVAT in one hour.

\subsection{Histology}

Visualization and identification of the type of fat within the PVAT of subclavian and iliac arteries was performed starting with the incubation of isolated PVAT specimens and rings with intact PVAT into $10 \%$ acetic zinc formalin overnight. Tissues were then processed using a Leica Benchtop Tissue Processor through the program shown in Table 1 for appropriate dehydration before embedding in paraffin wax. Thermo Shandon Histocentre 3 was used for the embedding of tissues in molten paraffin wax that subsequently solidified and blocks were stored in the freezer overnight. Thereafter, blocks of wax containing the tissue were sectioned into $7 \mu \mathrm{m}$ slices using Thermo Shandon Finesse 325 Manual Microtome and transferred onto slides for staining.

Table 1. Tissue processor program sequence of solutions and its corresponding times of incubation.

\begin{tabular}{cc}
\hline Solution & Time of Incubation \\
\hline $70 \%$ Ethanol & 2 Hours \\
$90 \%$ Ethanol & 2 Hours \\
$100 \%$ Ethanol contaning $2 \%$ Celloidin 1 & 2 Hours \\
$100 \%$ Ethanol contaning $2 \%$ Celloidin 2 & 2 Hours \\
$100 \%$ Ethanol contaning $2 \%$ Celloidin 3 & 2 Hours \\
Amyl Acetate 1 & 2 Hours \\
Amyl Acetate 2 & 2 Hours \\
Amyl Acetate 3 & 2 Hours \\
Molten wax 1 & 4 Hours \\
Molten wax 2 & 4 Hours \\
\hline
\end{tabular}


In order to stain the tissue, it must go through a process of rehydration. Therefore, slides were placed into Histoclear solution (a xylene substitute) for $10 \mathrm{mi}$ nutes to de-wax the tissues before the rehydration has begun. The slides were then processed through different concentrations of alcohol; 30 seconds in 100\% ethanol, 30 seconds in $90 \%$ ethanol and 1 minute in $70 \%$ ethanol; then the rehydration process was completed with washing for 1 minute in water. Thereafter, staining of tissue took place by submerging slides into Haematoxylin (Mayer's) stain for 8 minutes before washing with water for 1 minute. Slides were then submerged into Scott's solution for 1 minute to transform the red color of Haematoxylin to blue that stains the nucleus. Finally, two 1-minute washes with water took place before and after placing slides into $1 \%$ Eosin stain for 3 minutes. A further dehydration process was performed before mounting coverslips onto slides with DPX (histological mounting medium). Dehydration started with placing slides for 30 seconds in 70\% ethanol to rapidly differentiate Eosin, 1 minute in $90 \%$ ethanol to differentiate Eosin slowly, 4 minutes in 100\% ethanol and finally cleared in Histoclear solution for 10 minutes. Visualization and photographing of specimens were possible after allowing the DPX to dry overnight.

\subsection{Statistical Analysis}

Constriction and relaxation data were analyzed using two-way ANOVA test for the significance of the difference in response between rings with PVAT and rings without PVAT, while a student's paired t-test was performed for comparing NO secretion profile of PVAT before and after AICAR treatment. Resulting p-values are shown in each diagram and the cutoff for significance is the p-value of 0.05 , where values less than 0.05 are considered significant.

\section{Results}

\subsection{Constriction Data}

Subclavian and iliac rings with and without PVAT attached were tested with two vasoconstrictors, phenylephrine and noradrenaline; and responses to a range of concentrations were detected on the myograph in $\mathrm{mN}$ and used to construct concentration-response curves, in Figure 3, to demonstrate the effect of PVAT on constriction response.

Similar response curves can be seen in Figure 3(a) demonstrating that subclavian rings with and without PVAT attached contracted to the same extent to phenylephrine. The marginal differences in responses were in compliance with the two-way ANOVA test result of insignificance. Variability in responses seem to increase with the growing concentration of phenylephrine. On the other hand, the apparent difference between responses of rings with PVAT and of rings without PVAT to noradrenaline in Figure 3(b) was significant. The variability in responses was less in rings without PVAT than in rings with PVAT. Stronger contractions were produced by the subclavian rings with PVAT in response to noradrenaline compared to phenylephrine. The effects of phenylephrine on 


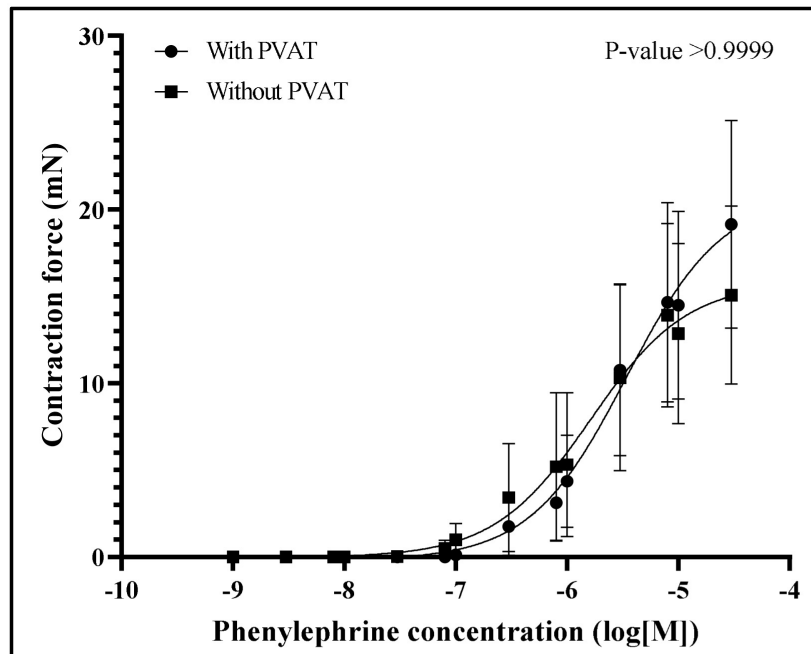

(a)

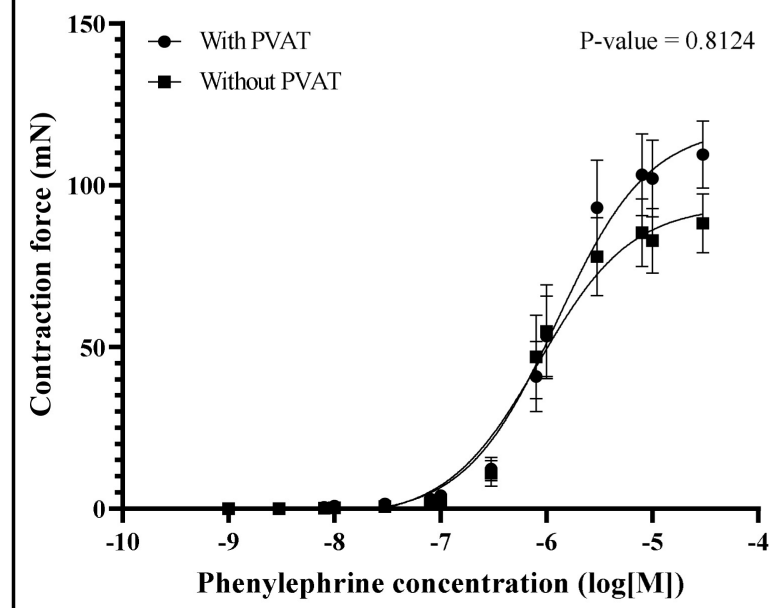

(c)

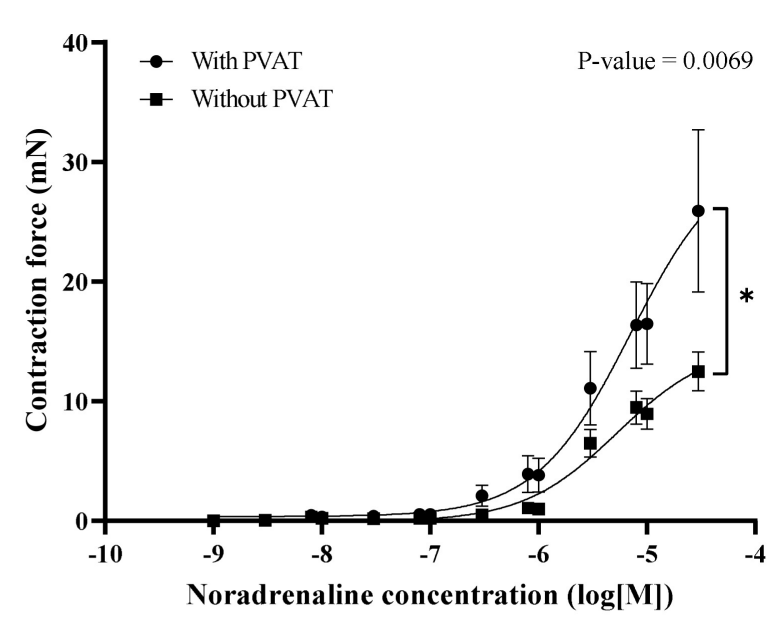

(b)

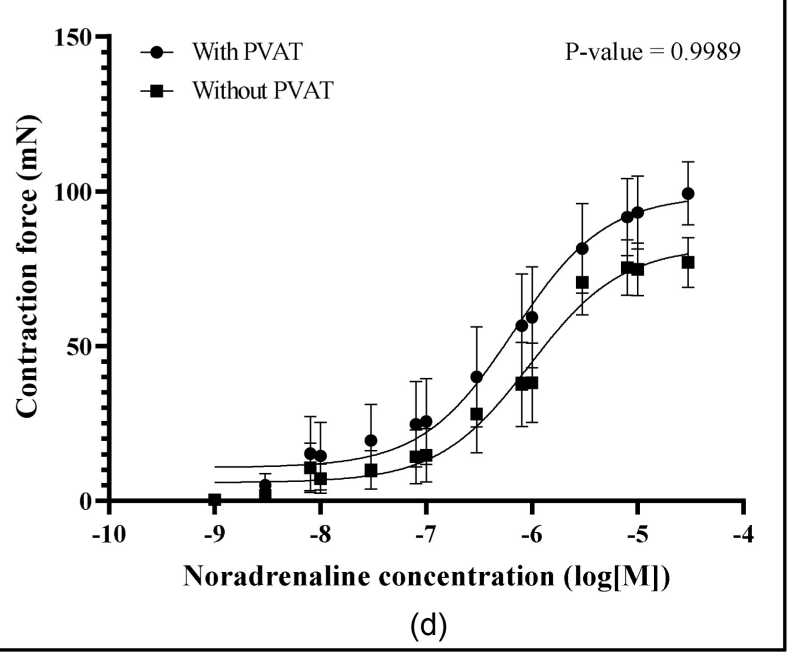

(d)

Figure 3. (a) and (b) show concentration-response curves of subclavian rings with and without PVAT attached ( $\mathrm{n}=6$ each) tested with cumulative dosing of phenylephrine and norepinephrine respectively. (c) and (d) show concentration-response curves of iliac rings with and without PVAT attached $(\mathrm{n}=6$ each) tested with cumulative dosing of phenylephrine and norepinephrine respectively. The asterisk $\left(^{\star}\right)$ identifies the significant difference.

subclavian rings appeared to be consistent with the effects on iliac rings. Iliac rings with and without PVAT appeared to have similar contraction responses to low and intermediate concentrations of phenylephrine, but rings with PVAT showed a stronger contraction to high concentrations ( $8 \mathrm{e}-6,1 \mathrm{e}-5$ and $3 \mathrm{e}-5)$ as displayed in Figure 3(c); nevertheless, the difference in response was deemed insignificant by the ANOVA test. The variability followed the same pattern as in the subclavian rings but maintained the level of stability in higher concentrations. Responses of iliac rings to noradrenaline seem to increase throughout the range of concentrations, with the rings with PVAT showing a stronger contraction as seen in Figure 3(d); however, the difference was statistically insignificant. 


\subsection{Relaxation Data}

Pre-contracted subclavian and iliac rings were treated with carbachol and SNAP to examine the effects of intact PVAT on endothelium-dependent and endothelium-independent relaxation respectively. Relaxation percentages in relation to relaxant concentrations have been used to construct concentration-response curves as shown in Figure 4.

Relaxation percentages of the subclavian rings treated with SNAP were higher in rings without PVAT as presented in Figure 4(b); but compared to rings with PVAT, the difference was statistically insignificant. However, the difference in relaxation percentages between rings with PVAT and rings without PVAT in the iliac artery was shown to be significant, as higher relaxation percentages were seen in rings without PVAT in Figure 4(d). Effects of carbachol on both subclavian and iliac arteries also produced higher relaxation percentages in rings

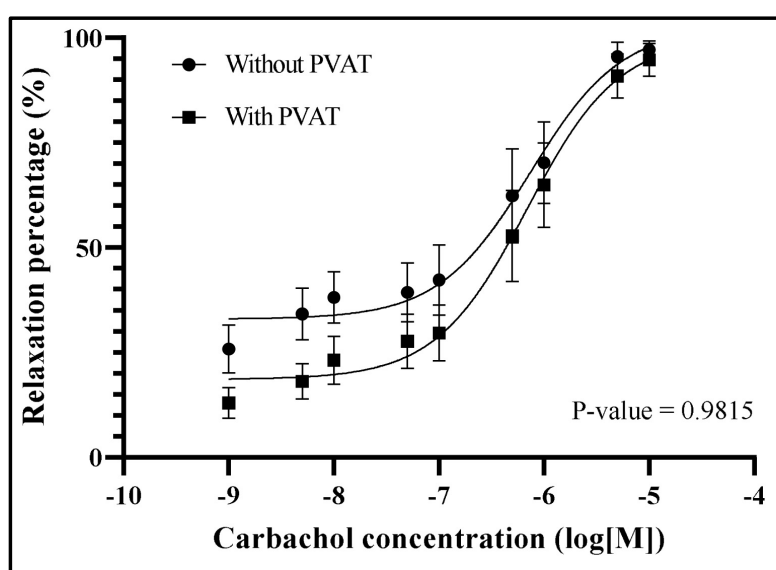

(a)

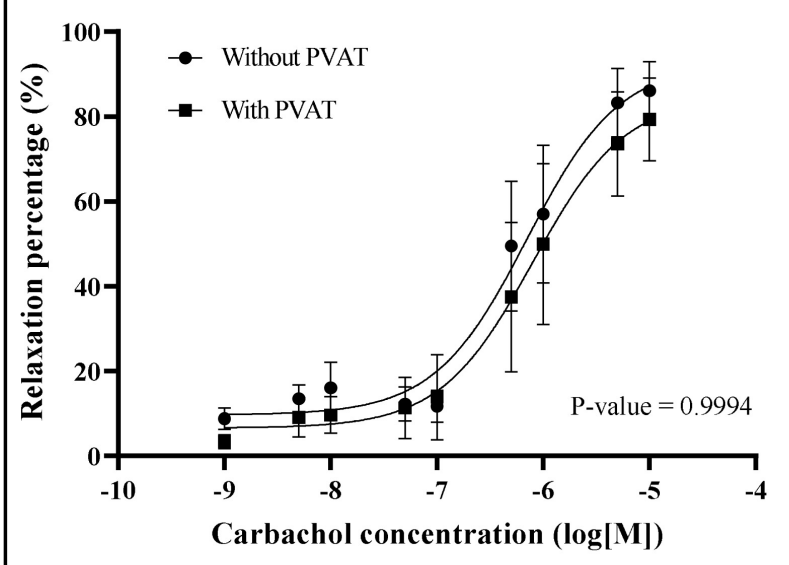

(c)

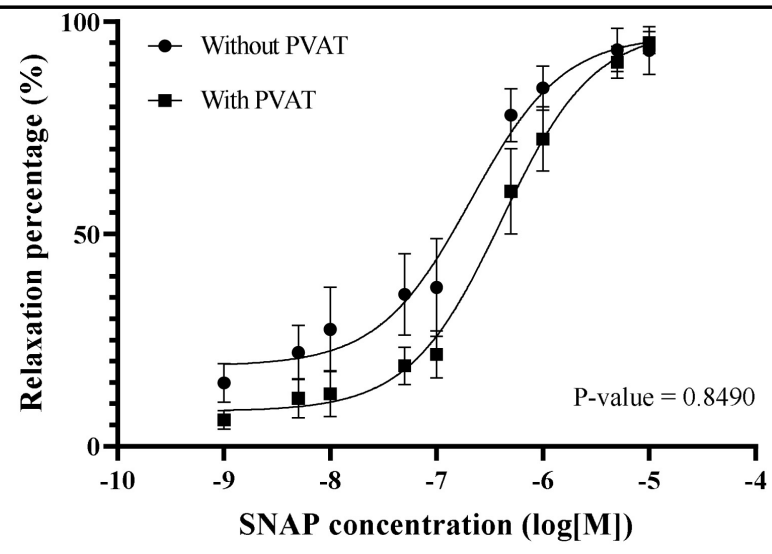

(b)

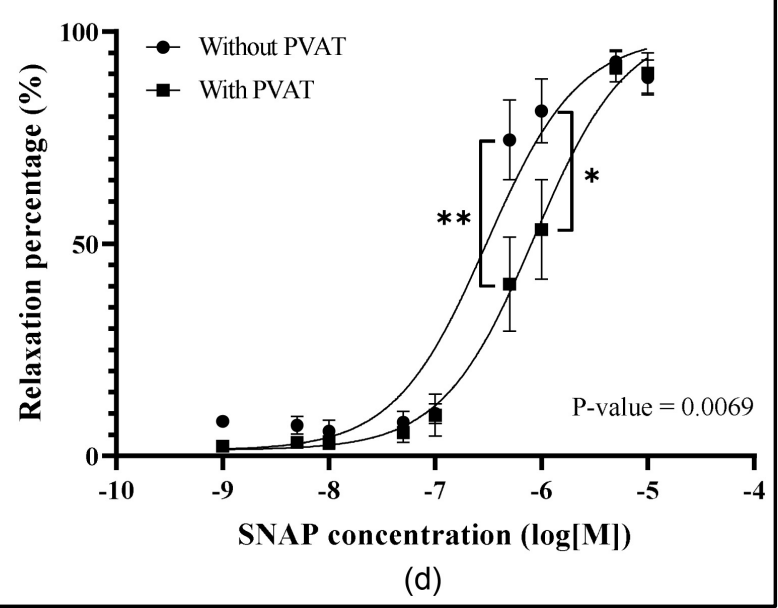

Figure 4. (a) and (b) show concentration-response curves of pre-contracted subclavian rings with and without PVAT attached ( $\mathrm{n}=6$ each) tested with cumulative dosing of carbachol and SNAP respectively. (c) and (d) show concentration-response curves of pre-contracted iliac rings with and without PVAT attached ( $\mathrm{n}=6 \mathrm{each}$ ) tested with cumulative dosing of carbachol and SNAP respectively. The asterisks $\left(^{*}\right)$ identify significant differences, greater significance is identified with more than one asterisk. 
without PVAT as seen in Figure 4(a) and Figure 4(c) respectively, but analysis of both differences in comparison to rings with PVAT were insignificant. Variability in responses of iliac rings with and without PVAT seem to overlap in almost all concentrations of carbachol. This data together with the constriction data provided above suggest that arteries with PVAT seem to have a tendency towards vasocontraction.

\subsection{Nitric Oxide Measurements}

Measurement of NO concentration by NOA was performed on eight samples of PVAT from each artery. The average of two concentration readings of each sample media was calculated and adjusted with subtraction of the average of two blank concentrations, in addition to multiplication by five to account for the previous dilution with methanol, and division by the weight of PVAT in media to obtain the NO secretion rate for every milligram of PVAT. Figure 5(a) and Figure 5(b) illustrate NO measurements for subclavian and iliac PVATs respectively.

Both subclavian and iliac PVAT demonstrated a higher secretion rate when treated with the AMPK-activating compound AICAR; however, secretion rates were insignificant in the case of iliac PVAT. At the same time, the difference in mean secretion rate between treated and untreated subclavian PVAT can be seen clearly in Figure 5(a), which was considered significant by the paired t-test. Contrary to subclavian PVAT, although the treated iliac PVAT produced an increased levels of NO per hour compared to the untreated PVAT as seen in Figure 5(b) below, the difference was insignificant.

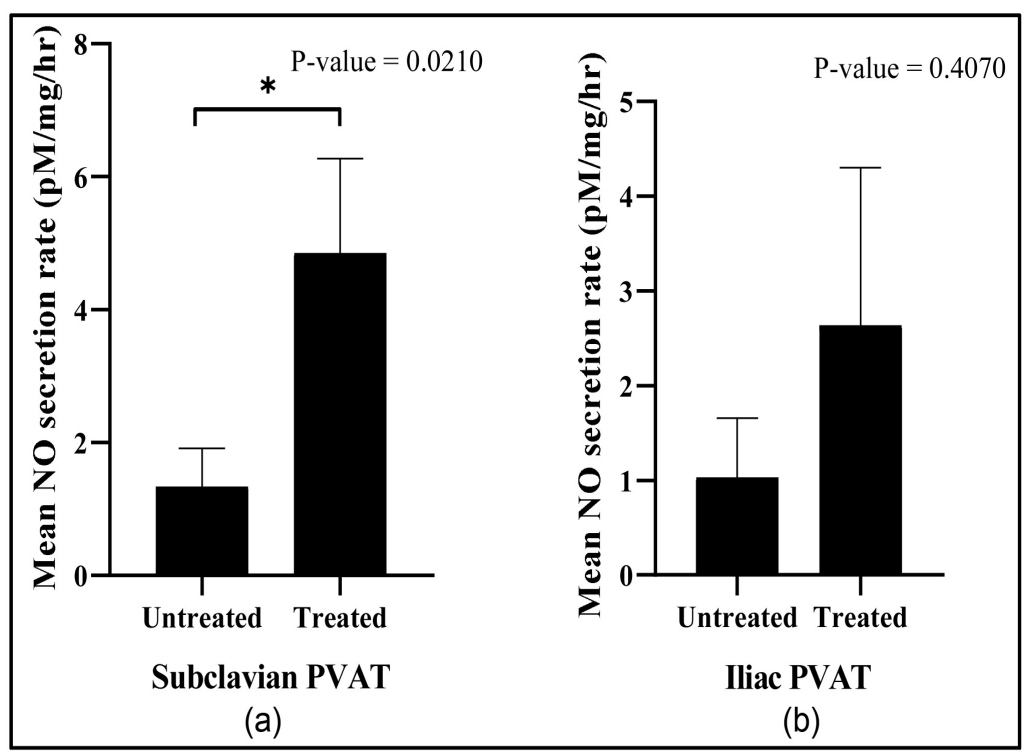

Figure 5. (a) Shows bar graph of the mean NO secretion rate for every milligram of subclavian PVAT treated and untreated with a concentration of $1 \mathrm{e}-3$ M of AICAR ( $n=8$ each). (b) Shows the same data for iliac PVAT. The asterisk $\left(^{*}\right)$ identifies the significant difference. 


\subsection{Histology Analysis}

After the inspection of stained sections of subclavian and iliac rings using the microscope, structural layers of the artery were identified and are shown in Figure 6 below.

Histological sections in Figure 6 shows the primary structural components of the arterial vessel. The red interior layer of the artery, seen in the far right of both (a) and (b), represents the endothelial cells with its nuclei recognized by the dark blue dots. Followed by a layer of smooth muscle cells that is clearly distinguished from its adjacent adventitia (Tunica externa) pink-colored layer. The outermost layer is the PVAT, where adipocytes and their nuclei look identical in both subclavian and iliac sections. The characteristic large lipid droplet of the adipocyte shown in (a) and (b) resemble lipid droplets of a WAT, suggesting that the subclavian and iliac PVAT carry properties of a white PVAT.

\section{Discussion}

The hallmark finding of this investigation is the marginal pro-contractile effects of subclavian and iliac PVAT seen in both functional tests, contraction and relaxation, performed using myography. Although mostly insignificant; difference in responses to both functional tests in both arteries are manifestations of many underlying factors that are related to the type, region and secretory profile of the PVAT; which might affect the degree of vascular response to a certain treatment under hypertensive conditions.

Activation of the alpha-adrenergic receptors located on the smooth muscle cells by phenylephrine and noradrenaline leads to depolarization of myocytes and ultimately contraction of the muscular bundle that leads to narrowing of the vascular wall [15]. There are many PVAT-derived molecules that may explain the increased contraction in rings with PVAT; however, leptin is a prominent

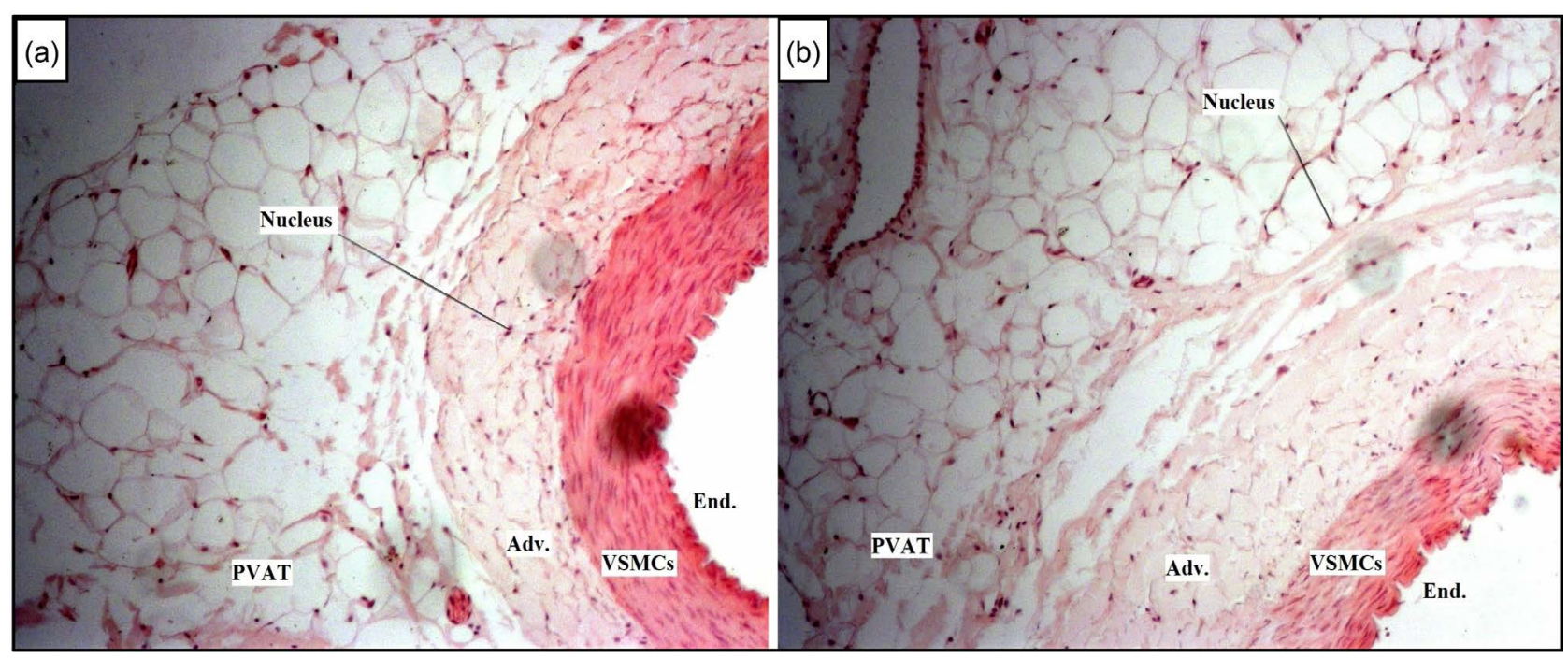

Figure 6. (a) Displays a stained section of the subclavian artery with its distinctive arterial layers, while (b) Displays the same for the iliac artery. PVAT, perivascular adipose tissue; Adv., adventitia; VSMCs, vascular smooth muscle cells; End, endothelium. 
molecule that has been suggested to exhibit contractile characteristics in many studies related to vascular modulation. In one study performed on rat portal vein (RPV), investigators found that stretching of RPV resulted in significant upregulation of leptin levels exceeding 100-fold, and a further increase in expression levels of leptin receptors by 10 -fold. The increased activity of leptin that led to vascular smooth muscle proliferation, evident by the increased weight of the tissue, was associated with increased activity of angiotensin II and endothelin-1 (ET-1) systems [16]. Moreover, endothelin-1 is a potent vasoconstrictor produced by the endothelium as a counterpart to NO. ET-1 mediates cell proliferation by activating ETA and ETB receptors and under pathological circumstances that lead to endothelial dysfunction, such as obesity, ET-1 secretion is highly augmented [17]. Another study has witnessed leptin-induced arterial hypertrophy by a different pathway. The investigation involved the use of swine coronary arteries exposed to obese concentrations of leptin. Results demonstrated that acute (30 minutes) and chronic (3 days) exposure to obese concentrations of leptin potentiated smooth muscle proliferation and increased contraction, which with inhibition of Rho kinase signaling were diminished. The use of fasudil, an Rho kinase inhibitor, produced a significant reduction in the $\mathrm{KCl}$-induced contractions of arteries treated with leptin relative to untreated arteries. The authors suggest that treatment with obese concentrations of leptin potentiated depolarization-induced contraction of coronary arteries; however, whether the activation of RhoA/Rho kinase is related to vascular activity of leptin is still unclear [18].

Another highly active vasoconstrictor that may account for the increased vascular contraction is angiotensin II. It is clear that the RAS is one of the most important regulators of the cardiovascular system and is becoming a substantial part of the endocrine function of the adipose tissue. Adipose tissue-derived RAS members angiotensin II and angiotensin 1 - 7 maintains physiological function of the vascular network and hence the cardiac function [19]. Many studies describe the alteration in adipose tissue secretion profile of RAS members in pathological conditions such as obesity and hypertension. In a study performed on rodents, researchers reported that overfeeding animals led to increased local secretion of angiotensinogen from adipocytes, which in turn resulted in the formation of high amounts of angiotensin II in the local adipose tissue. Further, angiotensin II was responsible for the upregulation of adipose tissue growth by stimulating prostacyclin release, which recruits preadipocytes and lipogenic enzymes. The increased adipose tissue mass and blood pressure of mouse models were evident for the activity of angiotensinogen. In addition, studies on obese hypertensive human subjects revealed elevated levels of angiotensin II in the adipose tissue that may be related to insulin resistance as blockade of RAS had beneficial impacts on the development of type 2 diabetes and insulin sensitivity [20]. Knowledge of the mechanisms by which adipose-tissue RAS induce insulin resistance under pathological metabolic conditions can impact standard treatments. In relation to the PVAT used in this investigation, the characteristic WAT 
content of rat mesenteric PVAT has been related to a significantly high expression levels of angiotensin II and its receptors $\mathrm{AT}_{1 \mathrm{a}}$ and $\mathrm{AT}_{2}$ compared to the brown periaortic PVAT in healthy conditions [21]. Lu and colleagues [10] have also reported a strong expression of angiotensinogen and angiotensin-converting enzyme (ACE) mRNA in rat mesenteric PVAT suggesting that white PVAT may have a higher sensitivity to angiotensin II. Subsequently, under the influence of conditions like obesity and hypertension, white visceral fat surrounding some major organs may affect its vascular networks.

In the present study, levels of PVAT-derived constricting factors may have overcome opposite actions of relaxing factors, such as adiponectin and angiotensin $1-7$, in relaxation functional testing with carbachol and SNAP. As was reported in Figure 5, NO levels were higher in PVAT treated with AICAR, with the subclavian PVAT levels being significant, suggesting that AMPK expression in PVAT is involved in generation of NO. However, functional testing with AICAR on pre-contracted rings with and without PVAT showed no relaxation in both rings (data not shown). This might be due to factors that could affect AMPK expression and function such as glucose, fatty acids and amino acids [22]. Moreover, NO generation by AMPK in the PVAT did not augment relaxation responses to carbachol and SNAP. Therefore, preliminary results suggest that carbachol and SNAP molecular pathways did not involve the activation of AMPK in NO production or subsequent activation of soluble guanylyl cyclase in VSMCs. Additionally, PVAT-derived relaxation factor adiponectin hypothesized to activate AMPK [8] may not have been secreted during relaxation. In addition, for the role of AMPK in endothelium-dependent and -independent relaxation to be discerned, further studies investigating the effects of AMPK inhibitors on precontracted rings during relaxation testing with carbachol and SNAP are warranted. Furthermore, investigating adiponectin expression levels in subclavian and iliac PVAT may account for the contractile actions of PVAT during relaxation tests. Generally, inhibition of angiotensin II may reveal different behavior by the arteries under contraction and relaxation functional testing.

Certainly, studies have reported the increased infiltration of immune cells into WAT under conditions like obesity, hypertension and diabetes, where recruitment rates of monocytes and activation of macrophages are increased, would eventually lead to activation of TNFs and interleukins that lead to inflammation [23]. Adipocyte hypertrophy also leads to elevated inflammatory signaling pathways, such as chemokines, oxidative stress and pro-inflammatory adipokines that attracts immune cells to the locally inflamed adipose tissue [2]. In a study comparing white PVAT of abdominal aorta to brown PVAT of thoracic aorta, mRNA of macrophage proinflammatory chemokines along with their receptors were reported to be significantly elevated in abdominal PVAT compared to thoracic PVAT under obese conditions in mice. Moreover, abdominal PVAT release of monocyte chemoattractant protein-1 (MCP-1) and macrophage recruitment were significantly higher than release in thoracic PVAT, with augmented levels 
seen in both PVATs under the influence of infused angiotensin II in obese mice. Incidents of abdominal aortic aneurysm were also measured and were found to be more frequent in angiotensin II-infused mice [24]. Another study investigating the activation and signaling of immune cells in white adipose tissue in humans, omental adipose tissue in obese individuals suffering from liver lipid accumulation showed an elevated expression of interleukin- 6 mRNA contrary to individuals with hyperinsulinemic-euglycemic status with glucose infusion. Additionally, high expression of interleukin- 6 cytokine correlated positively with white mesenteric adipose tissue lipolysis induced by interleukin- 6 signaling, which ultimately resulted in liver lipid accumulation and insulin resistance [25]. White adipose tissue is also related to development of atherosclerosis according to this study. It involved the comparison of apolipoprotein E (ApoE)-knockout mice with the wild type on high cholesterol diet and normal diet. Experimental results show increased levels of atherosclerotic plaques, macrophages and T-lymphocytes in white PVAT of aortic roots of AopE-knockout mice on cholesterol diet with increased transcription of interleukin- $1 \beta$, interleukin- 6 and interleukin-1 receptor. Moreover, liver staining of ApoE-knockout mice on cholesterol diet revealed high infiltration levels of macrophages and T-lymphocytes; as well as high levels of mRNA of interleukin-1 receptor. In ApoE-knockout mice on normal diet, white mesenteric adipose tissue showed increased levels of macrophages, mRNA of interleukin- 6 and interleukin-1; along with high plasma levels of TNF- $\alpha$ and interleukin-1 $\beta$ [26]. Therefore, with the growing evidence that white PVAT demonstrating increased infiltration of immune cells and cytokines in pathological states and the high expression of RAS members and its receptors, emphasis is projected towards identifying the molecular mechanisms that control the level of involvement of PVAT-induced adipokines, cytokines, interleukins and their corresponding enzymes in the aggravation of the pathological conditions, which may later prove the importance of PVAT in vascular modulation and the significant potential to be a pharmacological target in the near future.

\section{Conclusion}

The results of this investigation demonstrate the pro-contractile effects of subclavian and iliac PVAT under the influence of adrenergic receptor stimulation by phenylephrine and noradrenaline, and with relaxant factors carbachol and SNAP targeting endothelium-dependent and -independent mechanisms respectively. Histological staining revealed the WAT characteristics of the subclavian and iliac PVAT. Moreover, NO generation of PVAT treated with the AMPK activator AICAR was higher than untreated PVAT. Effects of AMPK may have been overcome by the pro-contractile effects of PVAT; however, to be certain of this, inhibitors of angiotensin II, leptin or anti-inflammatories may be used during functional testing of the rings. Additionally, measurements of ELISA levels of angiotensin II may account for the pro-contractility seen in PVAT. Although 
rabbits used were in healthy condition and without a sign of obesity, investigating immune cell activation and migration to PVAT along with expression levels of TNF- $\alpha$, interleukin-1, interleukin- 6 and macrophages may reveal obscure results. It is due to time limitations we were not able to deliver concrete data to fully prove our conclusions. Future studies may replicate the data shown using rabbit model and compare it to data from mouse model, as PVAT studies in rabbits were insufficient at the time this investigation was done.

\section{Conflicts of Interest}

The authors declare no conflicts of interest regarding the publication of this paper.

\section{References}

[1] Cheng, C.K., Bakar, H.A., Gollasch, M. and Huang, Y. (2018) Perivascular Adipose Tissue: The Sixth Man of the Cardiovascular System. Cardiovascular Drugs and Therapy, 32, 481-502. https://doi.org/10.1007/s10557-018-6820-z

[2] van Dam, A.D., Boon, M.R., Berbée, J.F.P., Rensen, P.C.N. and van Harmelen, V. (2017) Targeting White, Brown and Perivascular Adipose Tissue in Atherosclerosis Development. European Journal of Pharmacology, 816, 82-92. https://doi.org/10.1016/j.ejphar.2017.03.051

[3] Nosalski, R. and Guzik, T.J. (2017) Perivascular Adipose Tissue Inflammation in Vascular Disease. British Journal of Pharmacology, 174, 3496-3513. https://doi.org/10.1111/bph.13705

[4] Lian, X. and Gollasch, M. (2016) A Clinical Perspective: Contribution of Dysfunctional Perivascular Adipose Tissue (PVAT) to Cardiovascular Risk. Current Hypertension Reports, 18, 82. https://doi.org/10.1007/s11906-016-0692-Z

[5] Gil-Ortega, M., Somoza, B., Huang, Y., Gollasch, M. and Fernández-Alfonso, M.S. (2015) Regional Differences in Perivascular Adipose Tissue Impacting Vascular Homeostasis. Trends in Endocrinology \& Metabolism, 26, 367-375.

https://doi.org/10.1016/j.tem.2015.04.003

[6] Frontini, A. and Cinti, S. (2010) Distribution and Development of Brown Adipocytes in the Murine and Human Adipose Organ. Cell Metabolism, 11, 253-256. https://doi.org/10.1016/j.cmet.2010.03.004

[7] Boydens, C., Maenhaut, N., Pauwels, B., Decaluwé, K. and Van de Voorde, J. (2012) Adipose Tissue as Regulator of Vascular Tone. Current Hypertension Reports, 14, 270-278. https://doi.org/10.1007/s11906-012-0259-6

[8] Almabrouk, T.A.M., Ugusman, A.B., Katwan, O.J., Salt, I.P. and Kennedy, S. (2017) Deletion of AMPK $\alpha 1$ Attenuates the Anticontractile Effect of Perivascular Adipose Tissue (PVAT) and Reduces Adiponectin Release. British Journal of Pharmacology, 174, 3398-3410. https://doi.org/10.1111/bph.13633

[9] Thengchaisri, N. and Kuo, L. (2003) Hydrogen Peroxide Induces EndotheliumDependent and -Independent Coronary Arteriolar Dilation: Role of Cyclooxygenase and Potassium Channels. American Journal of Physiology-Heart and Circulatory Physiology, 285, H2255-H2263. https://doi.org/10.1152/ajpheart.00487.2003

[10] Lu, C., Su, L., Lee, R.M.K.W. and Gao, Y. (2010) Mechanisms for Perivascular Adipose Tissue-Mediated Potentiation of Vascular Contraction to Perivascular Neuronal Stimulation: The Role of Adipocyte-Derived Angiotensin II. European Journal 
of Pharmacology, 634, 107-112. https://doi.org/10.1016/j.ejphar.2010.02.006

[11] Takemori, K., Gao, Y., Ding, L., Lu, C., Su, L., An, W., Vinson, C. and Lee, R. (2007) Elevated Blood Pressure in Transgenic Lipoatrophic Mice and Altered Vascular Function. Hypertension, 49, 365-372. https://doi.org/10.1161/01.HYP.0000255576.16089.b9

[12] Virdis, A., Santini, F., Colucci, R., Duranti, E., Salvetti, G., Rugani, I., Segnani, C., Anselmino, M., Bernardini, N., Blandizzi, C., Salvetti, A., Pinchera, A. and Taddei, S. (2011) Vascular Generation of Tumor Necrosis Factor- $\alpha$ Reduces Nitric Oxide Availability in Small Arteries From Visceral Fat of Obese Patients. Journal of the American College of Cardiology, 58, 238-247. https://doi.org/10.1016/j.jacc.2011.01.050

[13] Lee, H., Després, J. and Koh, K.K. (2013) Perivascular Adipose Tissue in the Pathogenesis of Cardiovascular Disease. Atherosclerosis, 230, 177-184.

https://doi.org/10.1016/j.atherosclerosis.2013.07.037

[14] Daly, C.J. (2019) Examining Vascular Structure and Function Using Confocal Microscopy and 3D Imaging Techniques. In: Rea, P., Ed., Biomedical Visualisation, Advances in Experimental Medicine and Biology, Vol. 1120, Springer, Cham, 97-106. https://doi.org/10.1007/978-3-030-06070-1_8

[15] Reid, J.L. (1986) Alpha-Adrenergic Receptors and Blood Pressure Control. The American Journal of Cardiology, 57, E6-E12. https://doi.org/10.1016/0002-9149(86)90716-2

[16] Zeidan, A., Purdham, D.M., Rajapurohitam, V., Javadov, S., Chakrabarti, S. and Karmazyn, M. (2005) Leptin Induces Vascular Smooth Muscle Cell Hypertrophy through Angiotensin II- and Endothelin-1-Dependent Mechanisms and Mediates Stretch-Induced Hypertrophy. Journal of Pharmacology and Experimental Therapeutics, 315, 1075-1084. https://doi.org/10.1124/jpet.105.091561

[17] Marasciulo, F.L., Montagnani, M. and Potenza, M.A. (2006) Endothelin-1: The Yin and Yang on Vascular Function. Current Medicinal Chemistry, 13, 1655. https://doi.org/10.2174/092986706777441968

[18] Noblet, J.N., Goodwill, A.G., Sassoon, D.J., Kiel, A.M. and Tune, J.D. (2016) Leptin Augments Coronary Vasoconstriction and Smooth Muscle Proliferation via a RhoKinase-Dependent Pathway. Basic Research in Cardiology, 111, 25. https://doi.org/10.1007/s00395-016-0545-6

[19] Aghamohammadzadeh, R., Withers, S., Lynch, F., Greenstein, A., Malik, R. and Heagerty, A. (2012) Perivascular Adipose Tissue from Human Systemic and Coronary Vessels: The Emergence of a New Pharmacotherapeutic Target. British Journal of Pharmacology, 165, 670-682. https://doi.org/10.1111/j.1476-5381.2011.01479.x

[20] Engeli, S., Schling, P., Gorzelniak, K., Boschmann, M., Janke, J., Ailhaud, G., Teboul, M., Massiéra, F. and Sharma, A.M. (2003) The Adipose-Tissue Renin-Angiotensin-Aldosterone System: Role in the Metabolic Syndrome? The International Journal of Biochemistry \& Cell Biology, 35, 807-825.

https://doi.org/10.1016/S1357-2725(02)00311-4

[21] Gálvez-Prieto, B., Bolbrinker, J., Stucchi, P., de las Heras, A.I., Merino, B., Arribas, S., Ruiz-Gayo, M., Huber, M., Wehland, M., Kreutz, R. and Fernandez-Alfonso, M.S. (2008) Comparative Expression Analysis of the Renin-Angiotensin System Components between White and Brown Perivascular Adipose Tissue. Journal of Endocrinology, 197, 55-64. https://doi.org/10.1677/JOE-07-0284

[22] Jeon, S. (2016) Regulation and Function of AMPK in Physiology and Diseases. EXperimental \& Molecular Medicine, 48, e245. https://doi.org/10.1038/emm.2016.81

[23] Grant, R.W. and Dixit, V.D. (2015) Adipose Tissue as an Immunological Organ. 
Obesity, 23, 512-518. https://doi.org/10.1002/oby.21003

[24] Police, S.B., Thatcher, S.E., Charnigo, R., Daugherty, A. and Cassis, L.A. (2009) Obesity Promotes Inflammation in Periaortic Adipose Tissue and Angiotensin II-Induced Abdominal Aortic Aneurysm Formation. Arteriosclerosis, Thrombosis, and Vascular Biology, 29, 1458-1464. https://doi.org/10.1161/ATVBAHA.109.192658

[25] Wueest, S., Item, F., Lucchini, F.C., Challa, T.D., Müller, W., Blüher, M. and Konrad, D. (2016) Mesenteric Fat Lipolysis Mediates Obesity-Associated Hepatic Steatosis and Insulin Resistance. Diabetes, 65, 140-148. https://doi.org/10.2337/db15-0941

[26] Lohmann, C., Schäfer, N., von Lukowicz, T., Sokrates Stein, M.A., Borén, J., Rütti, S., Wahli, W., Donath, M.Y., Lüscher, T.F. and Matter, C.M. (2009) Atherosclerotic Mice Exhibit Systemic Inflammation in Periadventitial and Visceral Adipose Tissue, Liver, and Pancreatic Islets. Atherosclerosis, 207, 360-367.

https://doi.org/10.1016/j.atherosclerosis.2009.05.004 